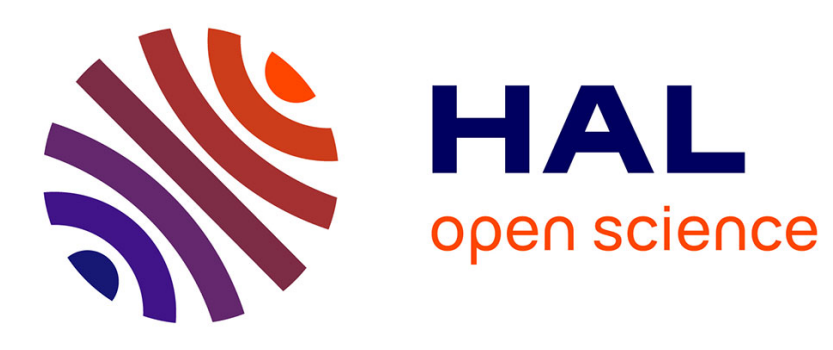

\title{
Proximity effect in parallelized microfabrication using two-photon polymerization
}

Luis Adrian Pérez Covarrubias, Caroline Arnoux, Quentin Carlier, Alexandre Khaldi, Patrice Baldeck, Kevin Heggarty

\section{- To cite this version:}

Luis Adrian Pérez Covarrubias, Caroline Arnoux, Quentin Carlier, Alexandre Khaldi, Patrice Baldeck, et al.. Proximity effect in parallelized microfabrication using two-photon polymerization. SPIE Photonics Europe 2020, Apr 2020, Online Only, France. pp.26, 10.1117/12.2555377 . hal-03104071

\section{HAL Id: hal-03104071 https://imt-atlantique.hal.science/hal-03104071}

Submitted on 26 May 2021

HAL is a multi-disciplinary open access archive for the deposit and dissemination of scientific research documents, whether they are published or not. The documents may come from teaching and research institutions in France or abroad, or from public or private research centers.
L'archive ouverte pluridisciplinaire $\mathbf{H A L}$, est destinée au dépôt et à la diffusion de documents scientifiques de niveau recherche, publiés ou non, émanant des établissements d'enseignement et de recherche français ou étrangers, des laboratoires publics ou privés. 


\title{
Proximity effect in parallelized microfabrication using two-photon polymerization
}

\author{
L. Pérez Covarrubias ${ }^{\mathrm{a}}$, C. Arnoux ${ }^{\mathrm{b}}$, Q. Carlier $^{\mathrm{a}}$, A. Khaldi ${ }^{\mathrm{a}}$, P. Baldeck ${ }^{\mathrm{b}}$, and K. Heggarty ${ }^{\mathrm{a}}$ \\ ${ }^{a}$ IMT-Atlantique, Optics Department, Technopole Brest-Iroise, 29285, BREST, France \\ buniversité de Lyon, ENS de Lyon, CNRS UMR 5182, Université Claude Bernard Lyon 1, \\ Laboratoire de Chimie, Lyon, France
}

\begin{abstract}
Two-photon polymerization is a photochemical process usually initiated by tightly focusing an ultrafast laser pulse into a volume of photosensitive photoresists with a high-numerical-aperture objective. Scanning a write "voxel" in 3D enables near free-form fabrication, but at a limited speed which is a critical factor for industrial purposes, because generally only a single writing-beam is used. Several strategies have been implemented to improve the fabrication speed, one such strategy is massive parallelization which is the approach used in our PHENOmenon H2020 European project. Massive parallelization can be realized by beam splitting diffractive optical elements which allow simultaneous fabrication with thousands of beams, decreasing the overall fabrication time. A major unexpected obstacle is encountered in massively parallelized fabrication: using several spots simultaneously to polymerize, local changes in the $2 \mathrm{PP}$ threshold have been observed. We linked this to the proximity effect. The aim of this study is to understand the proximity effect in parallel microfabrication using simulation to predict its behaviour and different systematic experiments to reduce the proximity effect such as changing photoresist, using thinner photoresist layers to increase oxygen penetration or using higher Numerical Aperture Objectives.
\end{abstract}

Keywords: Two-photon polymerization, diffractive optical element, massively parallel fabrication, photoresist, proximity effect, simulation.

\section{INTRODUCTION}

Two-photon polymerization (2PP) is a photochemical process usually initiated by tightly focusing an ultrafast laser pulse into a volume of photosensitive photoresist with a high-numerical-aperture objective. Scanning a write "voxel" in 3D enables near free-form fabrication, but at a limited speed which is a critical factor for industrial purposes, because only a single writing-beam is used. ${ }^{1}$

Different strategies have been implemented to improve fabrication speed, such as multi-beam interference, ${ }^{2}$ multi-focus ${ }^{3}$ and the recent work made by Saha et al. ${ }^{4}$ where the 3D structures were fabricated using a projectionbased layer-by-layer parallelization implemented with a micromirror array.

In our work in the PHENOmenon H2020 European project we show that massive parallelization can be realized by beam splitting diffractive optical elements (DOE) which allow simultaneous fabrication with thousands of beams, decreasing the overall fabrication time. A major unexpected obstacle has been encountered in massively parallelized fabrication. Using several spots simultaneously to polymerize, local changes in the $2 \mathrm{PP}$ threshold have been observed. We linked this to the presence of other light spots in the nearest neighbourhood of a studied light spot (spatial proximity effects) and/or to any previous exposure of the resist (whether producing polymerization or not) by spots scanned across the same plot area (temporal proximity effects). The proximity effect has been occasionally mentioned, for example Oakdale et al. ${ }^{5}$ and Saha et al. ${ }^{6}$ have reported its existence but in massive parallelization no records have been reported its specific effects.

In preliminary results obtained with parallel $2 \mathrm{PP}$ we faced difficulties to fabricate at high resolution because of overpolymerization in zones due to a specific proximity effects present in our system (See Figure 1.B) ${ }^{78}$. The proximity effect when performing parallel $2 \mathrm{PP}$ with a regular array of write beams can induce for example

Further author information: (Send correspondence to Luis A. Pérez Covarrubias)

E-mail: luis-adrian.perez-covarrubias@imt-atlantique.fr, Telephone: +33 (0)2 29001199 

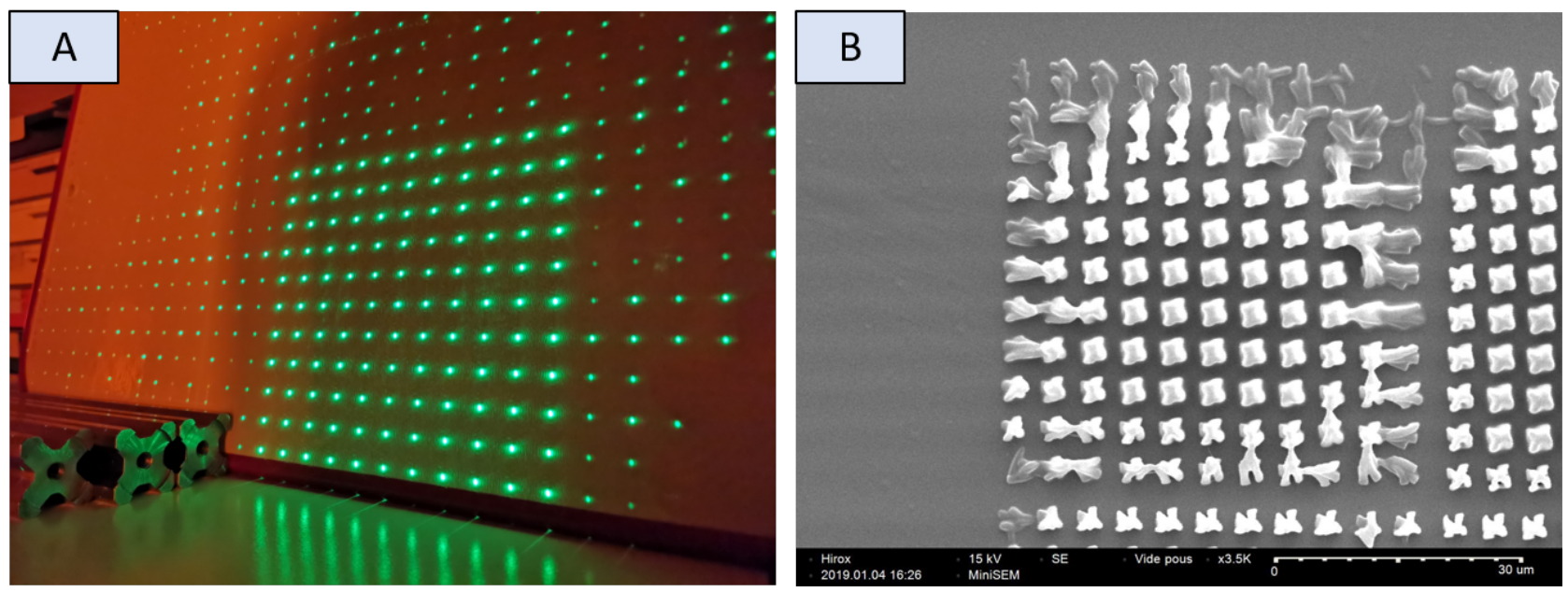

Figure 1. A) Example of a diffracted pattern from DOE 11x11 spot array and B) Observation of the proximity effect, experimental polymerized sample obtained with a DOE creating $11 \times 11$ laser spots.

undesired strong polymerization in the center of an array of spots, and/or reduced polymerization in the corners of the matrix of spots because there is less overlapping of exposure and/or oxygen depletion from neighboring spots.

We performed several studies to understand, quantify and reduce the proximity effect in own massively parallel write setup:

1) Calibration DOEs with the same number of spots (same write power) but varying inter-spot distances.

2) Use of different photoresists, both commercial and project-formulated, to compare the influence of their properties on the proximity effect.

3) Use of different microscope objectives with different Numerical Aperture (NA).

4) Use of different resist deposition techniques to control the Oxygen and radical diffusion.

5) Development of a phenomenological numerical model showing the $2 \mathrm{PP}$ thresholds spatially in matrices of spots. This is realized by summing in $3 \mathrm{D}$ the overlap produced by the point spread functions representing the light distribution of the individual spots in a spot array to investigate whether the local power density can reach a threshold level in undesired areas.

\section{EXPERIMENTAL SETUP AND METHODS}

The fabricated structures presented in this work were made in two different resist formulations. The first is based in the well-known commercial inorganic-organic resin OrmoComp®) (micro resist technology GmbH, Berlin, Germany) with the addition of 6 wt.- $\%$ commercial photo initiator (1,3,5-Tris(2-(9-ethylcabazyl-3)ethylene)benzene, Sigma Aldrich). The second formulation is based on a mixture of acrylate monomers with a photoinitiator designed by the chemistry laboratory of ENS in Lyon to improve the sensitivity of the photoresist in comparison with classical benchmarks.

Initially the resist deposition was made by drop casting as can be seen in figure 2.A and for large area resist deposition and to promote oxygen diffusion a "Doctor Blade" type Universal Applicator from Zehntner was used with a specific thickness, in our case layers of $50 \mu \mathrm{m}$ (See figure 2.B) . To perform the microfabrication experiments, a high pulse power laser with pulse duration of 400ps at $\lambda=532 \mathrm{~nm}$, frequency in $500 \mathrm{~Hz}$ and $35 \mu J$ per pulse was used. In the same manner, a home-made prototype laser optical set-up is used with an DOE into the optical path for replication of the write beam (See Figure 3). The DOEs were designed to display an array of NxN spots (See figure 1.A). The DOEs were fabricated in a layer of a positive photoresist coated on a glass substrate (S1805, MicroChem). ${ }^{9}$ In this work we used four DOEs with different spot separation (13 $\mu \mathrm{m}, 11.35$ $\mu m, 9.6 \mu m$ and $7.775 \mu m)$. 

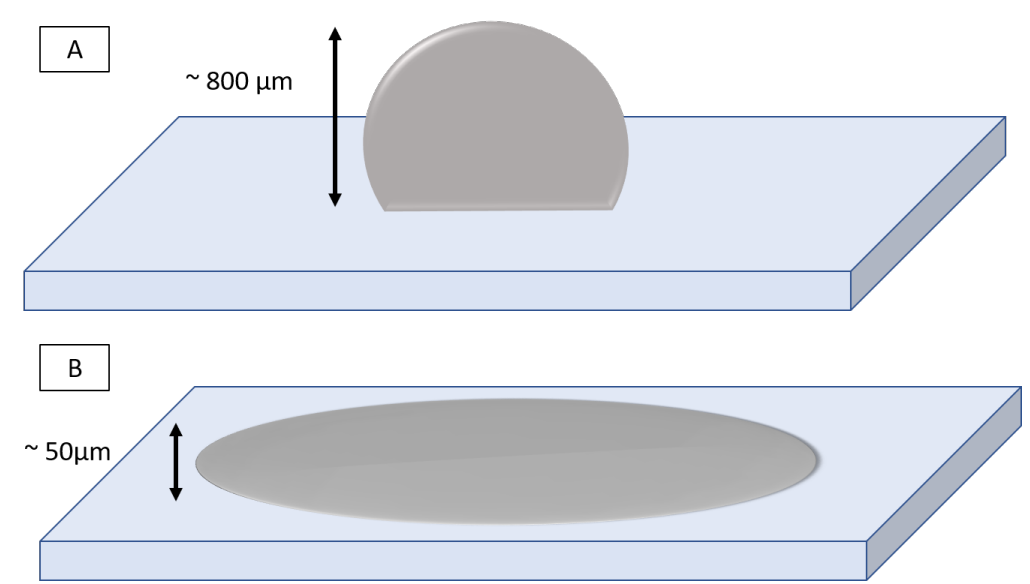

Figure 2. Photoresist deposition. A) Drop-cast deposition B) "Doctor Blade" deposition.

Two different objectives were used in this work: 1) A Zeiss "LD Plan-Neofluar" Korr Ph2 M27 40x and 0.6 (NA) with a Working distance (WD) of $2970 \mu m .2$ ) A Zeiss "Plan-Apochromat" Korr M27 40x and 0.95 (NA) with a Working Distance (WD) of $250 \mu \mathrm{m}$.

After fabrication, all structures were immersed in OrmoDevß developer purchased from Microresist Technology Gmbh (Berlin, Germany) and then photo-postcured. For characterization an optical microscope and a scanning electron microscope (SEM, Hirox) were used.

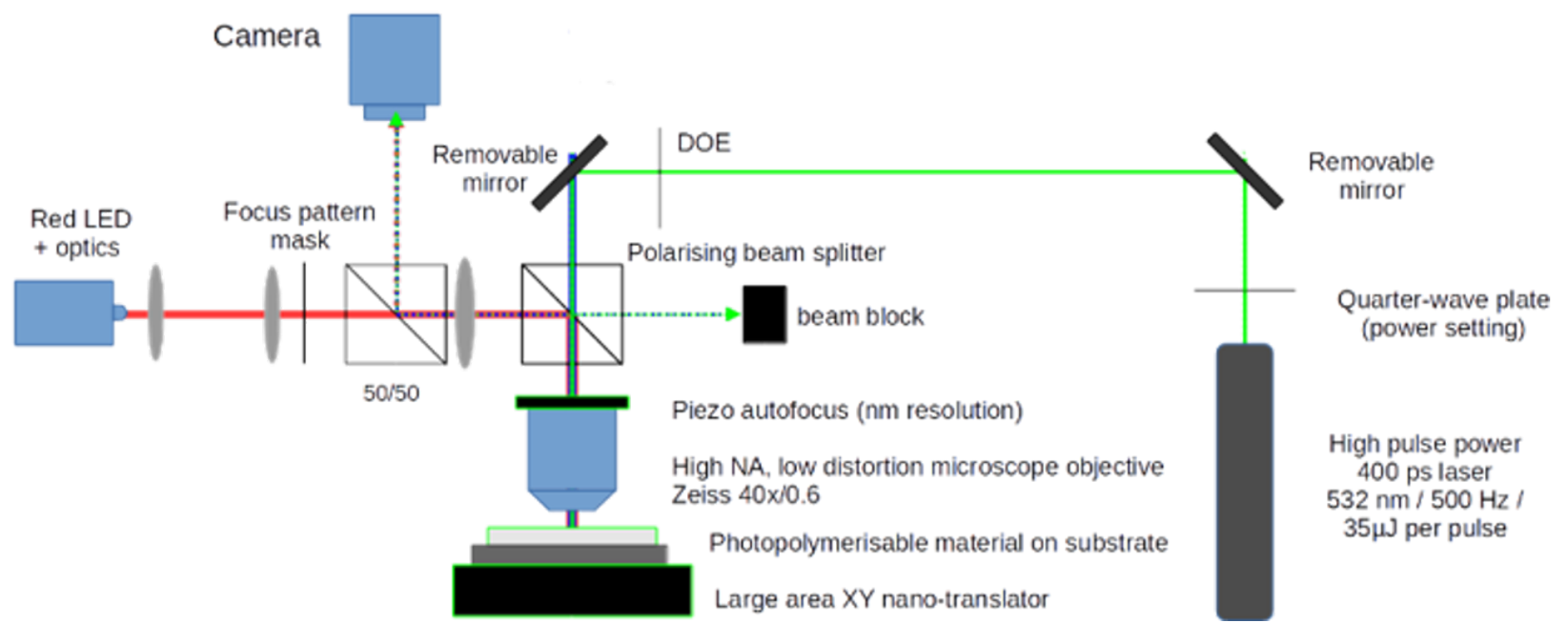

Figure 3. Optical setup used for microfabrication.

\section{NUMERICAL SIMULATIONS}

The development of a phenomenological numerical model was performed using the "PSF generator" and "Matlab" software. This is done by simulating the parallel-write process, performed, for instance, by summing in $3 \mathrm{D}$ the overlap produced by the point spread functions of the individual spots in a spot array to investigate whether the local power density can reach threshold level in undesired areas. In the numerical simulations the intensity was normalized to 1 , and any value above a fixed threshold value will be polymerized, in our case we arbitrarily fixed a threshold value.

Firstly, we simulated the point spread function (PSF) of an individual spot using a objective with NA of 0.6 Figure 4.A). Secondly, we summed the 3D PSF of individual spots in a spot array where the intensity distribution 
of 3X3 DOEs is simulated with same parameters ( See Figure 4.C). We observe the spatial proximity effect due to overlapping, where different polymerization areas are obtained between the central part and the corners of the array of spot.

In recent investigations we have found that a Gaussian-modelled radical diffusion process appears to be a promising technique for relating the simulation and observed experimental results. This part of the project is still under investigation to correctly adjust the experimental and simulation diffusion parameters.
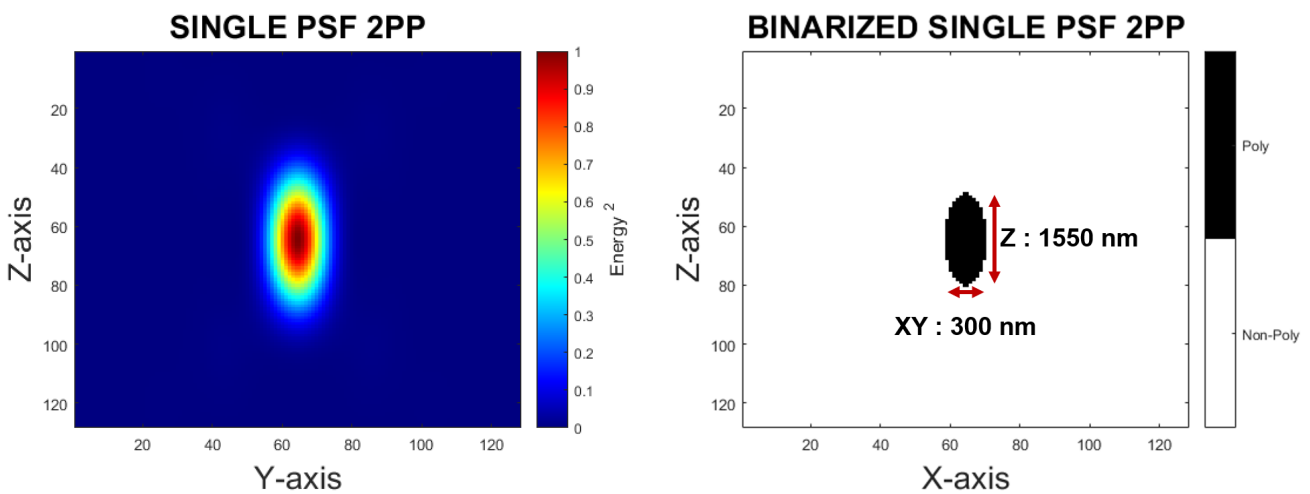

A) Side view. Numerical Aperture of 0.6
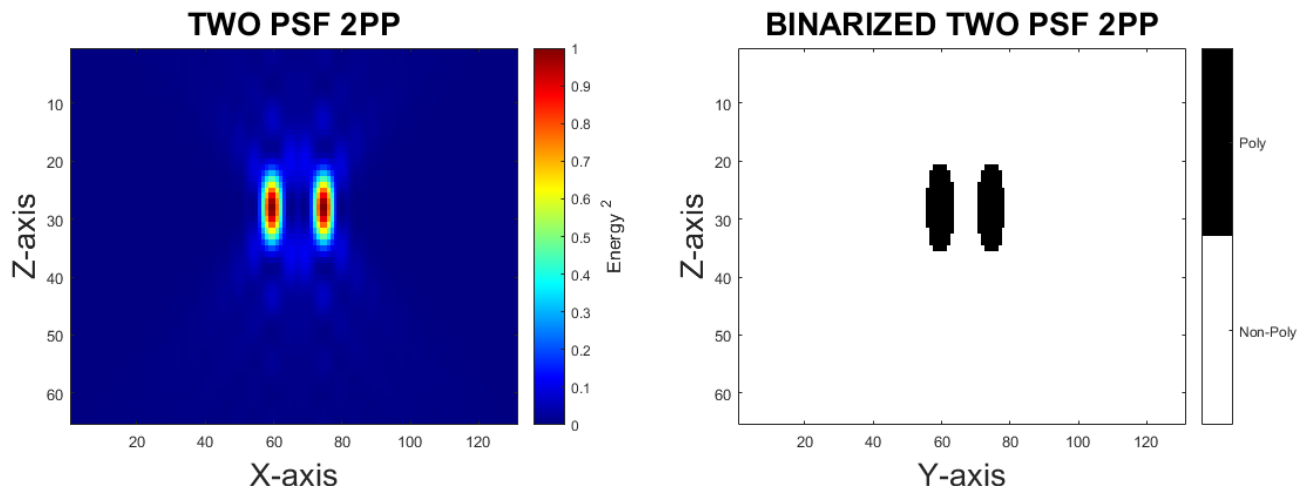

B) Side view. Numerical Aperture of 0.6.
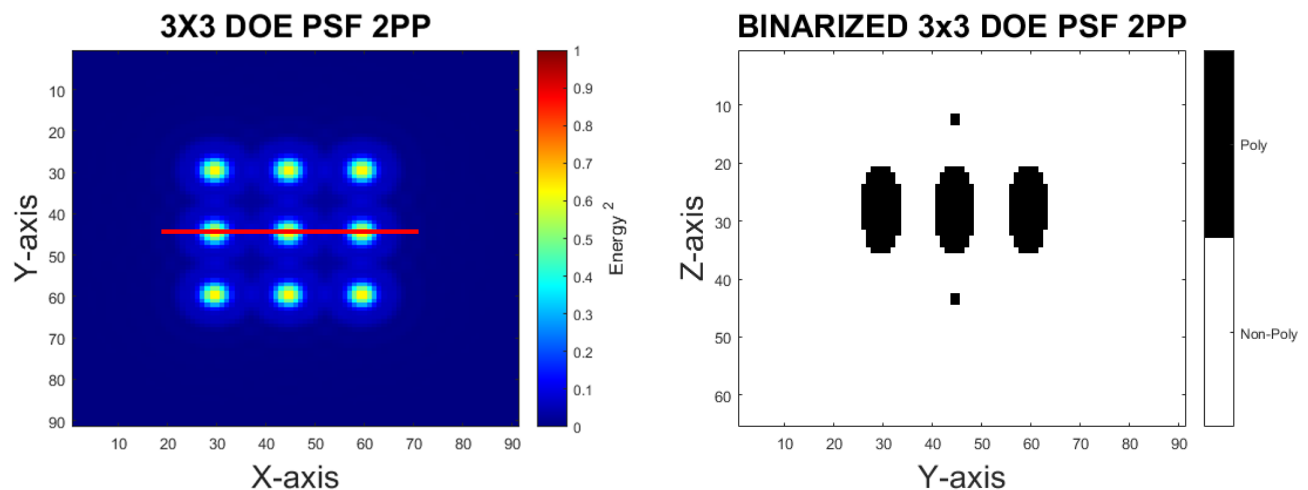

C) Top View and Side view. Numerical Aperture of 0.6 simulating a 3x3 DOE spot array.

Figure 4. Numerical simulation of single and overlapping point spread function keeping the same distance between spots (left: Point spread function. Right: Polymerized areas at a fixed threshold value). 


\section{EXPERIMENTAL RESULTS}

In the fabrication with 25 parallel laser beams using a 5x5 DOE with different spot separation and commercial photoresist, the overpolymerization due to the proximity effect in the center is stronger when the spot distance decreases probably because of the slow oxygen diffusion and high generation rate of radicals (See figure 5). This is a serious problem when we wish to fabricate structures with small features because the proximity effect will be stronger at smaller distances and the option to reduce the power is not possible because the corners and edges of the structure begin to disappear because they are not reaching the threshold (See figure 1.B).
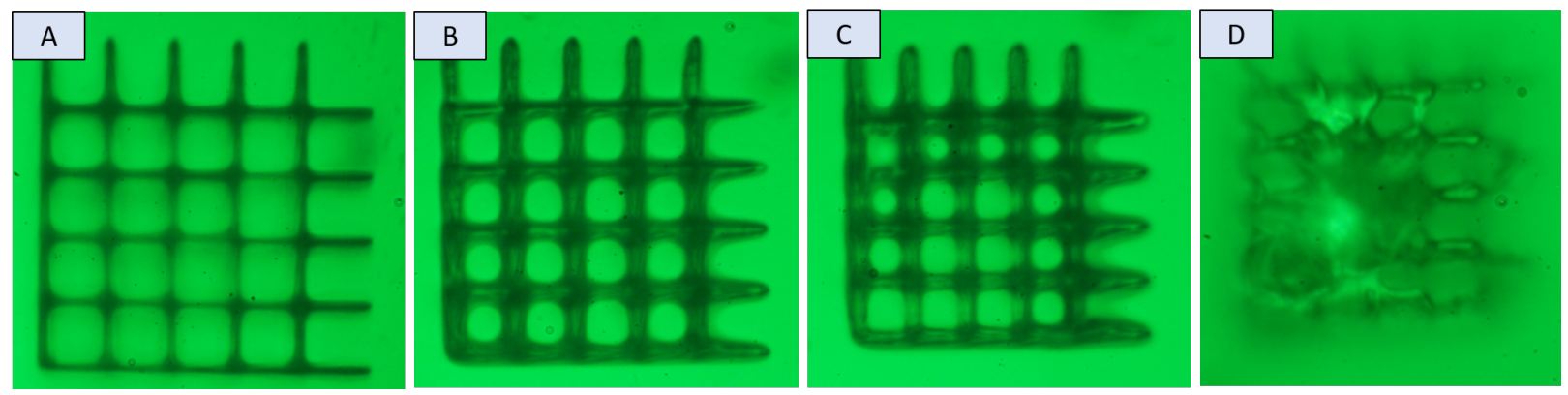

Figure 5. "L" shape patterns obtained by scanning a 5 X5 spot array. Identical laser power in each case but decreasing spot separation (13 $\mu \mathrm{m}, 11.35 \mu \mathrm{m}$, and $9.6 \mu \mathrm{m}, 7.775 \mu \mathrm{m}$ left to right).

In order to reduce the proximity effect we changed the standard fabrication parameters systematically such as the photoresist type, the deposition method and microscope objective. In figure 6.C, we observe a "L" shape structure obtained by scanning a 5X5 spot array with a spot separation of $9.6 \mu \mathrm{m}$, in this structure the standard parameters were used (OC+Cabazyl, Drop-cast and NA 0.6). The first change was to use the project-developed photoresist. The results show that using the this photoresist the proximity effect is weaker in the center than with the commercial photoresist, this is due to the enhanced photochemistry of the project developed photoresist which it was made specially for high resolution fabrication giving a stronger structure and better diffusion of radicals.(See figure $6 . \mathrm{A}$ ).

Following the same idea, we compared two different photoresist deposition methods for making thin layers. Drop-cast and "Dr. Blade" applicator were compared using the same photoresist. Figure 6.B demonstrates the benefit of using a "Dr. Blade" applicator because it helps deposit a homogeneous layer of $50 \mu m$ thickness which we presume helps promotes oxygen diffusion and local quenching during the fabrication more effectively than in a drop which has a thickness of hundreds of microns slowing the oxygen diffusion and provoking overpolymerization.

The comparison between different objectives is shown in figure 6.C \& D. The test plots were made at different write powers because the objective with NA of 0.95 concentrates light into a smaller volume. We observed that overpolymerization is weaker in the spot array center using the new objective presumably because the voxel is smaller.

Finally we show a structure using the best conditions (project-developed photoresist, thin layer using a "Dr. Blade" applicator and a higher NA objective), the structures are clean and do not present any observable proximity effect 6.E. These new parameters are helping us to fabricate 3D structures such as gratings, blazed gratings and more complex structures. 


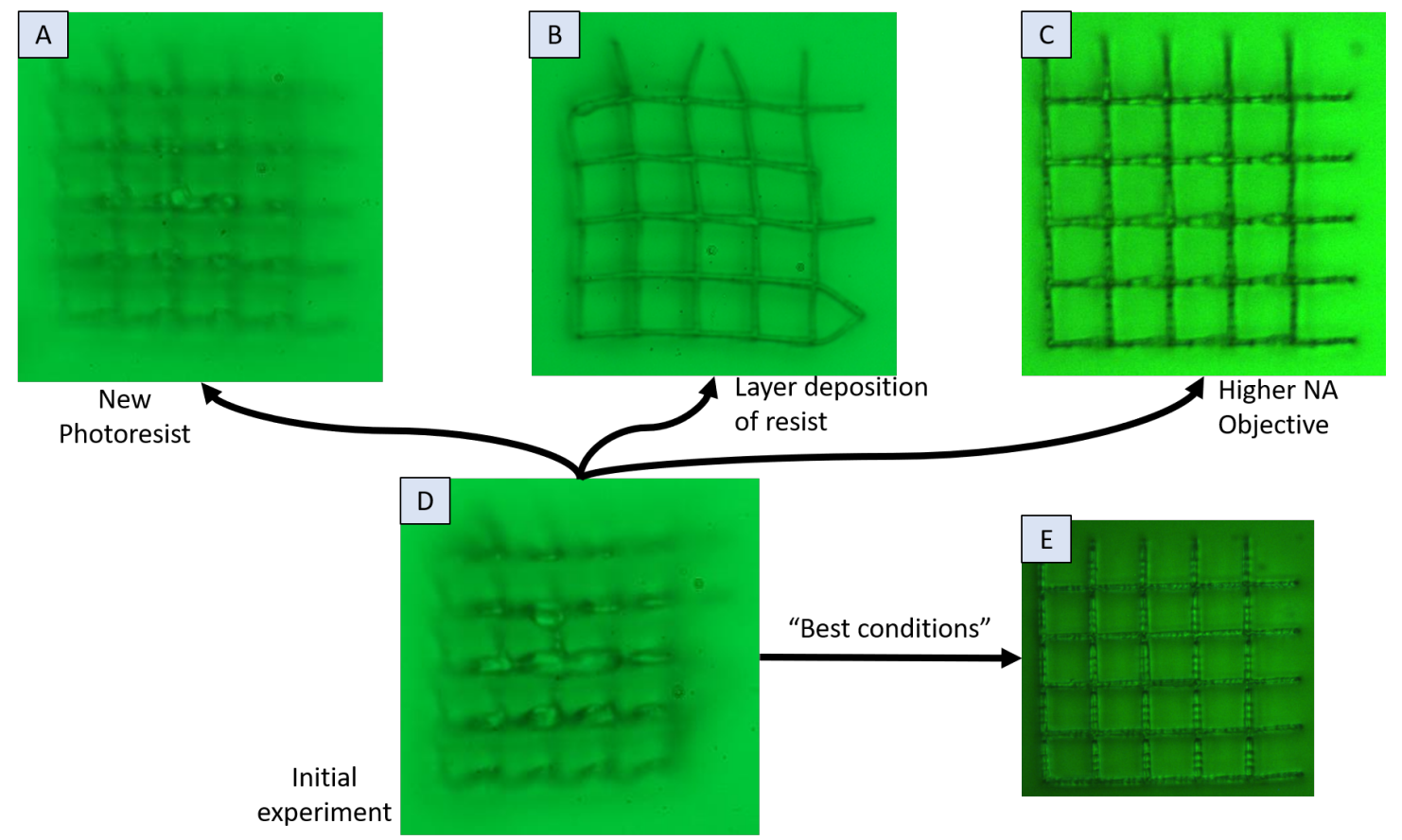

Figure 6. "L" shape patterns obtained by scanning a 5 X5 spot array with a spot separation of 9.6 $\mu m$. A) Project developed photoresist / Drop / NA 0.6. B) OC + Cabazyl PI / thin layer / NA 0.6. C) OC + Cabazyl PI / Drop / NA 0.95. D) OC + Cabazyl PI / Drop / NA 0.6. E) Project developed photoresist / thin layer / NA 0.95.

\section{CONCLUSION}

We have studied the proximity effect in parallel microfabrication, finding that it is a serious problem that can limit the fabrication resolution. The first step was to understand its behavior which allowed us to apply different techniques to reduce the effect such as high Numerical Aperture to increase resolution, deposition of resist in thin layers to promote oxygen diffusion and use of a photoresist than can improve sensitivity in comparison to classical benchmarks. We demonstrated that using these new conditions the proximity effect is reduced in the whole structure in parallel massive microfabrication. Further investigations in the modelling of the proximity effect continue to adapt the simulation parameters to experimental results. The study of the temporal proximity effect is under investigation to make a complete study of the phenomenon.

\section{ACKNOWLEDGMENTS}

This work has received funding from the European Union's Horizon 2020 research and innovation program under grant agreement $\mathrm{N}^{\circ}$ 780278. The dissemination of results herein reflects only the author's view and the European Commission is not responsible for any use that may be made of the information it contains.

\section{REFERENCES}

[1] Baldacchini, T., "Introduction," in [Three-Dimensional Microfabrication Using Two-photon Polymerization], Baldacchini, T., ed., Micro and Nano Technologies, xxiii - xxvi, William Andrew Publishing, Oxford (2016).

[2] He, C., Michael, S., and Arnold, G., "High-Efficiency Nanostructuring using Multi-Beam Interference by Consecutively Deposited Ultrashort Laser Pulses on Tool Steel," Journal of Laser Micro/Nanoengineering 13, 1-5 (Apr. 2018).

[3] Kato, J., Takeyasu, N., Adachi, Y., Sun, H.-B., and Kawata, S., "Multiple-spot parallel processing for laser micronanofabrication," (2005).

[4] Saha, S. K., Wang, D., Nguyen, V. H., Chang, Y., Oakdale, J. S., and Chen, S.-C., "Scalable submicrometer additive manufacturing," Science 366, 105-109 (Oct. 2019). 
[5] Oakdale, J. S., Smith, R. F., Forien, J.-B., Smith, W. L., Ali, S. J., Bayu Aji, L. B., Willey, T. M., Ye, J., van Buuren, A. W., Worthington, M. A., Prisbrey, S. T., Park, H.-S., Amendt, P. A., Baumann, T. F., and Biener, J., "Direct Laser Writing of Low-Density Interdigitated Foams for Plasma Drive Shaping," Advanced Functional Materials 27, 1702425 (Nov. 2017).

[6] Saha, S. K., Divin, C., Cuadra, J. A., and Panas, R. M., "Effect of Proximity of Features on the Damage Threshold During Submicron Additive Manufacturing Via Two-Photon Polymerization," Journal of Micro and Nano-Manufacturing 5, 031002 (Sept. 2017).

[7] Yang, L., Münchinger, A., Kadic, M., Hahn, V., Mayer, F., Blasco, E., Barner-Kowollik, C., and Wegener, M., "On the Schwarzschild Effect in 3D Two-Photon Laser Lithography," Advanced Optical Materials 7, 1901040 (Nov. 2019).

[8] Ligon, S. C., Husár, B., Wutzel, H., Holman, R., and Liska, R., "Strategies to reduce oxygen inhibition in photoinduced polymerization," Chemical Reviews 114, 557-589 (Jan. 2014).

[9] Kessels, M., Nassour, C., Grosso, P., and Heggarty, K., "Direct write of optical diffractive elements and planar waveguides with a digital micromirror device based uv photoplotter," Optics Communications - OPT COMMUN 283, 3089-3094 (08 2010). 